\title{
Dynamic Modeling and Control of a HexaRotor using Linear and Nonlinear Methods
}

\author{
Mostafa Moussid \\ Doctoral Student in Computer \\ Engineering \\ National Higher School of \\ Electricity and Mechanics
}

\author{
Adil Sayouti, PhD \\ Professor \\ School Royal Naval of Morocco \\ (ERN)
}

\author{
Hicham Medromi, PhD \\ Professor and Director \\ National Higher School of \\ Electricity and Mechanics
}

\begin{abstract}
A hexacopter aircraft is a class of helicopter, more specifically of multirotors. The hexacopter has several characteristics (mechanically simple, vertical takeoff and landing, hovering capacities, agile) that give it several operational advantages over other types of aircraft. But its beneficts come at a cost: the hexacopter has a highly nonlinear dynamics, coupled and underactuated which makes it impossible to operate without a feedback controller action.

In this work we present a detailed mathematical model for a Vertical Takeoff and Landing (VTOL) type Unmanned Aerial Vehicle (UAV) known as the hexarotor. The nonlinear dynamic model of the hexarotor is formulated using the Newton-Euler method, the formulated model is detailed including aerodynamic effects and rotor dynamics that are omitted in many literature. Three controls schemes, namely Proportional-DerivativeIntegral (PID) controller, backstepping and sliding mode (SMC), have been applied to control the altitude, attitude, heading and position of the hexacopter in space. Simulation based experiments were conducted to evaluate and compare the performance of three developed control techniques in terms of dynamic performance, stability and the effect of possible disturbances.
\end{abstract}

This article focuses on modeling strategy and command of a kind hexarotor type unmanned aerial vehicle (UAV). These developments are part of the overall project initiated by the team (EAS) of the Computer Laboratory, systems and renewable energy (LISER) of the National School of Electrical and Mechanical (ENSEM).

\section{Keywords}

Hexacopter; Vertical Takeo-and Landing; Nonlinear control; Newton-Euler method; PID; backstepping; sliding-mode;

\section{Nomenclature}

\begin{tabular}{|c|c|c|}
\hline Symbol & & Ieaning \\
\hline $\mathscr{R}_{I}$ & : & The earth inertial frame ( $\boldsymbol{R}_{I}$-frame) \\
\hline $\boldsymbol{R}_{B}$ & : & The body-fixed frame ( $\boldsymbol{R}_{B}$-frame $)$ \\
\hline $\mathrm{m} \epsilon \mathfrak{l}$ & : & Total mass of the hexa-rotor \\
\hline $\mathrm{g} \in \mathbb{R} \mathfrak{h}$ & : & Gravity constant \\
\hline$(\Phi, \theta, \psi) \in \mathfrak{l}^{3}$ & & Euler angle of rotorcraft \\
\hline$\xi \in \mathfrak{l}^{3}$ & : & $\begin{array}{l}\text { Position of the center of mass in the inertial } \\
\text { frame }\end{array}$ \\
\hline$\eta \in \mathfrak{h}^{3}$ & : & Position of the orientation in the body frame \\
\hline$v \in \mathfrak{h}^{3}$ & $\cdot$ & The translational velocity \\
\hline$\omega \in \mathfrak{l}$ & : & The angular velocity \\
\hline$K_{f a} \in \mathfrak{l i}^{3}$ & : & Propeller drag coefficient \\
\hline$\Omega_{\mathrm{r}} \in \mathfrak{R}$ & : & Overall propeller speed (rad/s) \\
\hline$J \in \mathfrak{l}^{3}$ & 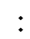 & The diagonal inertia matrix \\
\hline
\end{tabular}

$\begin{array}{lll}\mathrm{S}(\omega) \epsilon \mathfrak{R}^{3} & : & \text { Skew symmetric matrix } \\ J_{r} \in \mathfrak{R}^{3} & : & \text { Inertia for each rotor } \\ J_{x x / y y / z z} \in \mathfrak{i} & : & \text { The moments of inertia } \\ l \in \mathfrak{l} & : & \text { Distance to the center of gravity } \\ b \in \mathfrak{i} & : & \text { Thrust constant }\left[\mathrm{Ns}^{2}\right] \\ d \epsilon \mathfrak{i} & : & \text { The drag factor }[\mathrm{Nms}] . \\ \left(k_{p}, k_{i}, k_{d}\right) \in \mathfrak{h}^{3}: & \text { Proportional, integral and derivative gain }\end{array}$

\section{INTRODUCTION}

This work will focus on the modeling and control of a hexarotor type UAV. The reason for choosing the hexarotor is in addition to its advantages (their increased load capacity and high maneuverability.etc.), the research field is still facing some challenges in the control field because the hexarotor is a highly nonlinear, multivariable system and since it has six Degrees of Freedom but only four actuators, it is an under actuated system [1].

Under-actuated systems are those having a less number of control inputs compared to the system's degrees of freedom. They are very difficult to control due to the nonlinear coupling between the actuators and the degrees of freedom [2]. Although the most common flight control algorithms found in literature are linear flight controllers, these controllers can only perform when the hexarotor is flying around hover, they suffer from huge performance degradation whenever the hexarotor leaves the nominal conditions or performs aggressive maneuvers [3].

The contributions of this work are: deriving an accurate and detailed mathematical model of the hexarotor UAV, developing linear and nonlinear control algorithms and applying those on the derived mathematical model in computer based simulations. The work will be concluded with a comparison between the developed control algorithms in terms of their dynamic performance and their ability to stabilize the system under the effect of possible disturbances.

The paper remainder is organized as follows. In the next Section the mathematical formulation and the dynamic model of the hexacopter are described, while the applications of three different control techniques PID, Backstepping and Slidingmode to hexarotor are presented in section III. In section IV, the simulation results are given to highlight the proposed method, while conclusion is drawn in the last section V.

\section{DYNAMIC MODEL OF THE HEXAROTOR}

The mathematical model of the hexacopter has to describe its attitude according to the well-known geometry of this UAV. More specifically, this aerial vehicle basically consists of six propellers located orthogonally along the body frame. Figure 1 
shows this configuration. There are three movements that describe all possible combinations of attitude: Roll (rotation around the $\mathrm{X}$ axis) is obtained when the balance of rotors 1,2 and 3 (or 6, 5 and 4) is changed (speed increases or decreases). By changing the angle, lateral acceleration is obtained; pitch movement (rotation around the $\mathrm{Y}$ axis) is obtained when the balance of the speed of the rotors 1 and 6 (or 3 and 4) is changed. The angle change results in a longitudinal acceleration; yaw (rotation about the $\mathrm{Z}$ axis) is obtained by a simultaneous change of speed of the motors $(1,3,5)$ or $(2,4,6)$.

\subsection{Hexacopter Kinematics}

This subsection describes the dynamical models of the Six Rotor. The schematic structure of the hexacopter and the rotational directions of the propellers are illustrated in Figure 1. In order to describe the hexacopter motion only two reference systems are necessary: earth inertial frame ( -frame) and bodyfixed frame $\left(\boldsymbol{R}_{B}\right.$-frame).

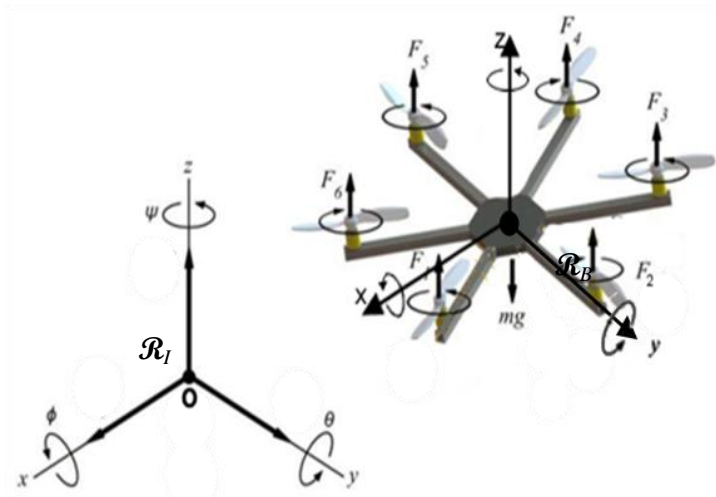

Fig 1: The structure of hexarotor and its frames

The orientation of the hexacopter is given by the three Euler angles, namely yaw angle $\psi$, pitch angle $\theta$ and roll angle $\phi$ that together form the vector $\eta=[\phi, \theta, \psi]^{T} .(\phi$ and $\theta \epsilon]-\frac{\pi}{2}, \frac{\pi}{2}[; \psi \epsilon$ ]$-\pi, \pi[$ )

The position of the vehicle in the inertial frame is given by the vector $\xi=[\mathrm{x}, \mathrm{y}, \mathrm{z}]^{T}$.

So, the transformation related to the position and the angular velocity from the body to the inertial frame is obtained respectively by:

$\mathfrak{R}_{I}^{B}=\left(\begin{array}{ccc}\cos \theta \cos \psi & \cos \psi \sin \theta \sin \phi-\sin \psi \cos \phi & \cos \psi \sin \theta \cos \phi+\sin \psi \sin \phi \\ \sin \psi \cos \theta & \sin \theta \sin \psi \sin \phi+\cos \psi \cos \theta & \cos \phi \sin \psi \sin \theta-\cos \psi \sin \phi \\ -\sin \theta & \sin \phi \cos \theta & \cos \theta \cos \phi\end{array}\right)$

$$
\text { And } \boldsymbol{R}_{r}=\left[\begin{array}{ccc}
1 & 0 & -\sin \theta \\
0 & \cos \phi & \sin \phi \cos \theta \\
0 & -\sin \phi & \cos \phi \cos \theta
\end{array}\right] \quad \text { where } \omega=\mathscr{R}_{r} \dot{\eta}
$$

\section{2 Applied forces and torques}

The two main forces come from gravity and the thrust of the rotors but to make the model more realistic rotor drag and air friction is also included. The UAV rotorcraft system are quite complex. Their movements are governed by several effects either mechanical or aerodynamic. The main effects on the acting hexarotor have been listed in the following table:
Table 1. The main effects on the acting hexarotor

\begin{tabular}{|l|l|}
\multicolumn{1}{|c|}{ Effects } & \multicolumn{1}{|c|}{ Fountainhead } \\
\hline Aerodynamics effects & Rotating propellers \\
\hline Inertiel counter torque & Speed change of propellers \\
\hline Effect of gravity & Position of the center of mass \\
\hline Gyroscopic effects & Change in the direction of the drone \\
\hline L'effet de frottement & Tous les mouvements du drone \\
\hline
\end{tabular}

In order to get equations of motion of entire system, the following assumptions have been made:

- The hexacopter is a rigid body;

- The hexacopter has a symmetrical structure;

- Tensions in all directions are proportional to the square of the propeller speed.

To derive the dynamic model of the hexacopter (position and attitude); the Newton-Euler formalism is used [4]. Therefore the following equations are obtained:

$$
\left[\begin{array}{cc}
m I_{3 \times 3} & 0_{3 \times 3} \\
0_{3 \times 3} & J
\end{array}\right]\left[\begin{array}{c}
\dot{V} \\
\dot{\omega}
\end{array}\right]+\left[\begin{array}{cc}
\omega & \wedge m V \\
\omega & \wedge J \omega
\end{array}\right]=\left[\begin{array}{c}
\sum \mathrm{F} \\
\sum \mathrm{M}
\end{array}\right]
$$

\subsubsection{Forces}

- Gravity force: $\mathrm{F}_{\mathrm{g}}=\left[\begin{array}{lll}0 & 0 & -\mathrm{mg}\end{array}\right]^{T}$.

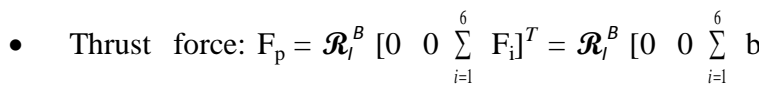
$\left.\omega_{i}^{2}\right]^{T}$

- $\quad$ Rotor drag: $\mathrm{F}_{\mathrm{t}}=\mathrm{k}_{\mathrm{ft}} v=I_{3 \times 3}\left[\begin{array}{lll}\mathrm{k}_{\mathrm{ftx}} & \mathrm{k}_{\mathrm{fty}} & \mathrm{k}_{\mathrm{ftz}}\end{array}\right]^{T} \mathrm{x} \dot{\xi}$

The vector of the drag forces, $\mathrm{k}_{\mathrm{ft}}=\operatorname{diag}\left(\mathrm{k}_{\mathrm{ftx}}, \mathrm{k}_{\mathrm{fty}}, \mathrm{k}_{\mathrm{ftz}}\right)$.

- Air resistance : $\mathrm{T}_{\mathrm{i}}=C_{t} \rho A r^{2} \Omega_{i}^{2}=\mathrm{d} \Omega_{i}^{2}$

Where A is a blade area, $\rho$ the density of air, $r$ the radius of the blade and $\Omega$ i the angular velocity of a propeller.

\subsubsection{Torques}

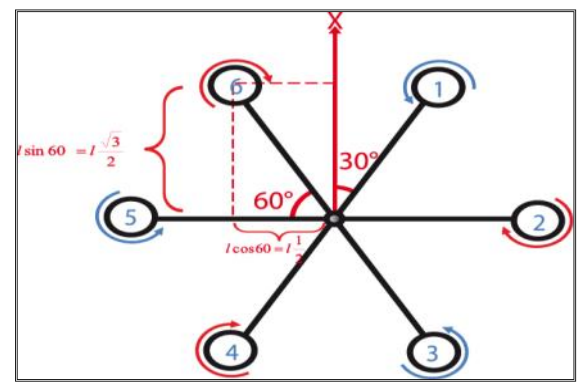

Fig 2: Hexacopter Rotor Distances to Center of Gravity

- Actuator action :

$\checkmark \quad$ Roll torque: $\boldsymbol{M}_{\mathrm{x}}=$

$b l\left(-\Omega_{2}^{2}+\Omega_{5}^{2}+\frac{1}{2}\left(-\Omega_{1}^{2}-\Omega_{3}^{2}+\Omega_{4}^{2}+\Omega_{6}^{2}\right)\right)$ 
$\checkmark$ Pitch torque: $\boldsymbol{M}_{\mathrm{r}}=b l \frac{\sqrt{3}}{2}\left(-\Omega_{1}^{2}+\Omega_{3}^{2}+\Omega_{4}^{2}-\Omega_{6}^{2}\right)$

$\checkmark$ Yaw torque: $\boldsymbol{M}_{\mathrm{z}}=d\left(-\Omega_{1}^{2}+\Omega_{2}^{2}-\Omega_{3}^{2}+\Omega_{4}^{2}-\Omega_{5}^{2}+\Omega_{6}^{2}\right)$

The vector $\boldsymbol{M}_{\mathrm{f}}$ is defined as: $\boldsymbol{M}_{\mathrm{f}}=\left[\begin{array}{lll}\boldsymbol{M}_{\mathrm{x}} & \boldsymbol{M}_{\mathrm{y}} & \boldsymbol{M}_{\mathrm{z}}\end{array}\right]^{T}$

- Torque aerodynamique resistance :

$$
\boldsymbol{M}_{\mathrm{a}}=K_{f a} \omega^{2}=\left[K_{f a x} \dot{\phi}^{2} K_{f a y} \dot{\theta}^{2} K_{f a z} \dot{\psi}^{2}\right]^{T}
$$

- gyroscopic effect from Propeller:

$$
\boldsymbol{M}_{\mathrm{gh}}=\sum_{i=1}^{6} \Omega_{\mathrm{r}} \wedge J_{r}\left[\begin{array}{lll}
0 & 0 & (-1)^{\mathrm{i}+1} \omega_{i}
\end{array}\right]^{T}
$$

- Yaw counter torque : $\boldsymbol{M}_{\mathrm{gh}}=\left[0,0, J_{r} \dot{\Omega}_{r}\right]^{T}$

With $\Omega_{r}=-\Omega_{1}+\Omega_{2}-\Omega_{3}+\Omega_{4}-\Omega_{5}+\Omega_{6}$

\subsubsection{Hexacopter mathematical model}

The equations of motion, that governs the translational and the rotational motion for the hexarotor with respect to the body frame are

\subsubsection{Translational dynamic}

$$
\begin{gathered}
m \dot{\xi}=\sum \mathcal{F}=F_{p}+F_{g}+F_{t} \\
\ddot{\mathrm{y}}=1 / \mathrm{m}(\cos \phi \sin \theta \sin \psi-\sin \phi \cos \psi)\left(\sum_{i=1}^{6} \mathrm{~F}_{\mathrm{i}}\right)-\mathrm{kfty} \dot{\mathrm{y}} / \mathrm{m} \\
\ddot{\mathrm{z}}=1 / \mathrm{m}(\cos \theta \cos \phi)\left(\sum_{i=1}^{6} \mathrm{~F}_{\mathrm{i}}\right)-\mathrm{kftz} \dot{\mathrm{z}} / \mathrm{m}-\mathrm{g}
\end{gathered}
$$

\subsubsection{Rotational dynamics}

$$
\left\{\begin{array}{c}
J \dot{\omega}=-\omega \wedge J \omega+\boldsymbol{M}_{\mathrm{f}}-\boldsymbol{M}_{\mathrm{a}}-\boldsymbol{M}_{\mathrm{gh}} \\
J_{x x} \ddot{\phi}=\dot{\theta} \dot{\psi}\left(J_{y y}-J_{z z}\right)-K_{f a x} \dot{\phi}^{2}-J_{r} \Omega_{\mathrm{r}} \dot{\theta}+ \\
+b l\left(-\Omega_{2}^{2}+\Omega_{5}^{2}+\frac{1}{2}\left(-\Omega_{1}^{2}-\Omega_{3}^{2}+\Omega_{4}^{2}+\Omega_{6}^{2}\right)\right) \\
J_{y y} \ddot{\theta}=\dot{\phi} \dot{\psi}\left(J_{z z}-J_{x x}\right)-K_{f a y} \dot{\theta}^{2}+J_{r} \Omega_{\mathrm{r}} \dot{\phi}+ \\
+b l \frac{\sqrt{3}}{2}\left(-\Omega_{1}^{2}+\Omega_{3}^{2}+\Omega_{4}^{2}-\Omega_{6}^{2}\right) \\
J_{z z} \ddot{\psi}=\dot{\phi} \dot{\theta}\left(J_{x x}-J_{y y}\right)-K_{f a z} \dot{\psi}^{2}+ \\
+d\left(-\Omega_{1}^{2}+\Omega_{2}^{2}-\Omega_{3}^{2}+\Omega_{4}^{2}-\Omega_{5}^{2}+\Omega_{6}^{2}\right)
\end{array}\right.
$$

The hexacopter's total thrust force and torque control inputs $\mathrm{u}_{1}$, $\mathrm{u}_{2}, \mathrm{u}_{3}, \mathrm{u}_{4}$ are related to the six motor's speed by the following equations: $\mathrm{U}^{T}=\left[\mathrm{u}_{1}, \mathrm{u}_{2}, \mathrm{u}_{3}, \mathrm{u}_{4}\right]$ is the vector of (artificial) input variables[5]:

$$
\left[\begin{array}{l}
u_{1} \\
u_{2} \\
u_{3} \\
u_{4}
\end{array}\right]=\left[\begin{array}{cccccc}
b & b & b & b & b & b \\
\frac{-b l}{2} & -b l & \frac{-b l}{2} & \frac{b l}{2} & b l & \frac{b l}{2} \\
\frac{-b l \sqrt{3}}{2} & 0 & \frac{b l \sqrt{3}}{2} & \frac{b l \sqrt{3}}{2} & 0 & \frac{-b l \sqrt{3}}{2} \\
-d & d & -d & d & -d & d
\end{array}\right]\left[\begin{array}{c}
\Omega_{1}^{2} \\
\Omega_{2}^{2} \\
\Omega_{3}^{2} \\
\Omega_{4}^{2} \\
\Omega_{5}^{2} \\
\Omega_{6}^{2}
\end{array}\right]
$$

If the rotor velocities are needed to be calculated from the control inputs, an inverse relationship between the control inputs and the rotors' velocities is needed, which can be acquired by inverting the matrix in (4) to give,

$$
\left\{\begin{array}{l}
\Omega_{1}^{2}=\frac{1}{6 b l}\left(l \mathrm{u}_{1}+2 \mathrm{u}_{2}-\frac{\mathrm{b} l}{d} \mathrm{u}_{4}\right) \\
\Omega_{2}^{2}=\frac{1}{6 b l}\left(l \mathrm{u}_{1}+\mathrm{u}_{2}-\sqrt{3} \mathrm{u}_{3}+\frac{\mathrm{b} l}{d} \mathrm{u}_{4}\right) \\
\Omega_{3}^{2}=\frac{1}{6 b l}\left(l \mathrm{u}_{1}-\mathrm{u}_{2}-\sqrt{3} \mathrm{u}_{3}-\frac{\mathrm{b} l}{d} \mathrm{u}_{4}\right) \\
\Omega_{4}^{2}=\frac{1}{6 b l}\left(l \mathrm{u}_{1}-2 \mathrm{u}_{2}+\frac{\mathrm{b} l}{d} \mathrm{u}_{4}\right) \\
\Omega_{5}^{2}=\frac{1}{6 b l}\left(l \mathrm{u}_{1}-\mathrm{u}_{2}+\sqrt{3} \mathrm{u}_{3}-\frac{\mathrm{b} l}{d} \mathrm{u}_{4}\right) \\
\Omega_{6}^{2}=\frac{1}{6 b l}\left(l \mathrm{u}_{1}+\mathrm{u}_{2}+\sqrt{3} \mathrm{u}_{3}+\frac{\mathrm{b} l}{d} \mathrm{u}_{4}\right)
\end{array}\right.
$$

\subsubsection{Total system model}

Finally, this derivation provides the $2^{\text {nd }}$ order differential equations for the aircraft's position and orientation in space. Applying relation (1) to (5) and rewriting the matrix equation in from of system, we obtain the following:

$$
\begin{aligned}
& \left\{\begin{array}{l}
\ddot{\phi}=\frac{1}{J_{x x}}\left[\dot{\theta} \dot{\psi}\left(J_{y y}-J_{z z}\right)-K_{f a x} \dot{\phi}^{2}-J_{r} \Omega_{\mathrm{r}} \dot{\theta}+\mathrm{u}_{2}\right] \\
\ddot{\theta}=\frac{1}{J_{y y}}\left[\dot{\phi} \dot{\psi}\left(J_{z z}-J_{x x}\right)-K_{f a y} \dot{\theta}^{2}+J_{r} \Omega_{\mathrm{r}} \dot{\phi}+\mathrm{u}_{3}\right] \\
\ddot{\psi}=\frac{1}{J_{z z}}\left[\dot{\phi} \dot{\theta}\left(J_{x x}-J_{z z}\right)-K_{f a z} \dot{\psi}^{2}+\mathrm{u}_{4}\right] \\
\ddot{\mathrm{x}}=-\frac{\mathrm{kftx}}{m} \dot{\mathrm{x}}+\frac{1}{m} \mathrm{u}_{\mathrm{x}} \mathrm{u}_{1} \\
\ddot{\mathrm{y}}=-\frac{\mathrm{kfty}}{m} \dot{\mathrm{y}}+\frac{1}{m} \mathrm{u}_{\mathrm{y}} \mathrm{u}_{1} \\
\ddot{\mathrm{z}}=-\frac{\mathrm{kftz}}{m} \dot{\mathrm{z}}-\mathrm{g}+\frac{\cos \phi \cos \theta}{m} \mathrm{u}_{1}
\end{array}\right. \\
& \text { with : }\left\{\begin{array}{l}
\mathrm{u}_{\mathrm{x}}=\cos \phi \cos \psi \sin \theta+\sin \phi \sin \psi \\
\mathrm{u}_{\mathrm{y}}=\cos \phi \sin \theta \sin \psi-\sin \phi \cos \psi
\end{array}\right.
\end{aligned}
$$

The dynamic model presented in equation set (6) can be rewritten in the state-space form $\dot{\mathrm{X}}=f(X, U) . \mathrm{X} \in \mathfrak{h}^{12}$ is the vector of state variables given as follows: 


$$
\begin{aligned}
& \mathrm{X}^{T}=\left[\begin{array}{llllllllllll}
\phi & \dot{\phi} & \theta & \dot{\theta} & \psi & \dot{\psi} & \mathrm{x} & \dot{x} & \mathrm{y} & \dot{y} & \mathrm{z} & \dot{z}
\end{array}\right] \\
& \mathrm{x}_{12} \text { ] } \\
& \begin{array}{l|l}
\mathrm{x}_{1}=\phi & \mathrm{x}_{7}=\mathrm{x} \\
\mathrm{x}_{2}=\dot{x}_{1}=\dot{\phi} & \mathrm{x}_{8}=\dot{x}_{7}=\dot{x} \\
\mathrm{x}_{3}=\theta & \mathrm{x}_{9}=\mathrm{y} \\
\mathrm{x}_{4}=\dot{x}_{3}=\dot{\theta} & \mathrm{x}_{10}=\dot{x}_{9}=\dot{y} \\
\mathrm{x}_{5}=\psi & \mathrm{x}_{11}=\mathrm{z} \\
\mathrm{x}_{6}=\dot{x}_{5}=\dot{\psi} & \mathrm{x}_{12}=\dot{x}_{11}=\dot{z}
\end{array} \\
& \left\{\begin{array}{l}
\dot{x}_{2}=\ddot{\phi}=\mathrm{a}_{1} \mathrm{x}_{4} \mathrm{x}_{6}+\mathrm{a}_{2} x_{2}^{2}+\mathrm{a}_{3} \Omega_{\mathrm{r}} \mathrm{x}_{4}+\mathrm{b}_{1} \mathrm{u}_{2} . \\
\dot{x}_{4}=\ddot{\theta}=\mathrm{a}_{4} \mathrm{x}_{2} \mathrm{x}_{6}+\mathrm{a}_{5} x_{4}^{2}+\mathrm{a}_{6} \Omega_{\mathrm{r}} \mathrm{x}_{2}+\mathrm{b}_{2} \mathrm{u}_{3} . \\
\dot{x}_{6}=\ddot{\psi}=\mathrm{a}_{7} \mathrm{x}_{2} \mathrm{x}_{4}+\mathrm{a}_{8} x_{6}^{2}+\mathrm{b}_{3} \mathrm{u}_{4} . \\
\dot{x}_{8}=\ddot{x}=\mathrm{a}_{9} \mathrm{x}_{8}+\frac{1}{m} \mathrm{u}_{\mathrm{x}} \mathrm{u}_{1} . \\
\dot{x}_{10}=\ddot{y}=\mathrm{a}_{10} \mathrm{x}_{10}+\frac{1}{m} \mathrm{u}_{\mathrm{y}} \mathrm{u}_{1} . \\
\dot{x}_{12}=\ddot{z}=\mathrm{a}_{11} \mathrm{x}_{12}+\frac{\cos \phi \cos \theta}{m} \mathrm{u}_{1}-\mathrm{g} .
\end{array}\right.
\end{aligned}
$$

To simplify, define,

$$
\begin{array}{c|c|c}
a_{1}=\left(J_{y y}-J_{z z}\right) / J_{x x} & a_{2}=-K_{f a x} / J_{x x} & a_{9}=-K_{f t x} / \mathrm{m} \\
a_{4}=\left(J_{z z}-J_{x x}\right) / J_{y y} & a_{5}=-K_{f a y} / J_{y y} & a_{10}=-K_{f t y} / \mathrm{m} \\
a_{7}=\left(J_{x x}-J_{y y}\right) / J_{z z} & a_{8}=-K_{f a z} / J_{z z} & a_{11}=-K_{f t z} / \mathrm{m} \\
a_{3}=-J_{l} / J_{x x} & a_{6}=-J_{l} / J_{y y} & \\
b_{1}=l / J_{x x} & b_{2}=l / J_{y y} & b_{3}=l / J_{z z}
\end{array}
$$

Rewriting the last equation (7) to have the angular accelerations in terms of the other variables (Rotational equation of motion),

$$
\left(\begin{array}{c}
\dot{x}_{2} \\
\dot{x}_{4} \\
\dot{x}_{6}
\end{array}\right]=\left[\begin{array}{l}
\mathrm{a}_{1} \mathrm{x}_{4} \mathrm{x}_{6}+\mathrm{a}_{2} x_{2}^{2}+\mathrm{a}_{3} \Omega_{\mathrm{r}} \mathrm{x}_{4}+\mathrm{b}_{1} \mathrm{u}_{2} \\
\mathrm{a}_{4} \mathrm{x}_{2} \mathrm{x}_{6}+\mathrm{a}_{5} x_{4}^{2}+\mathrm{a}_{6} \Omega_{\mathrm{r}} \mathrm{x}_{2}+\mathrm{b}_{2} \mathrm{u}_{3} \\
\mathrm{a}_{7} \mathrm{x}_{2} \mathrm{x}_{4}+\mathrm{a}_{8} x_{6}^{2}+\mathrm{b}_{3} \mathrm{u}_{4}
\end{array}\right]
$$

With the choice of the control input vector $U$, it is clear that the rotational subsystem is fully-actuated, it is only dependant on the rotational state variables $\mathrm{x} 1$ to $\mathrm{x} 6$ that correspond to $\phi, \dot{\phi}, \theta, \dot{\theta}$, $\psi, \dot{\psi}$ respectively.

Rewriting the last equation (7) to have the accelerations in terms of the other variables, we get translational equation of motion,

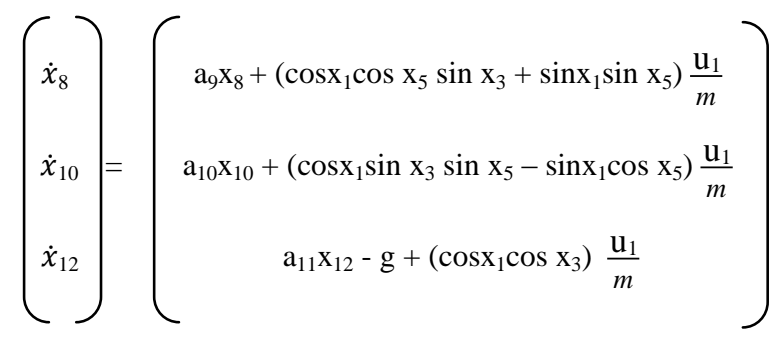

It is clear here that the translational subsystem is under_ actuated as it dependant on both the translational state variables and the rotational ones.

It is worthwhile to note in the latter system that the angles and their time derivatives do not depend on translation components. On the other hand, the translations depend on the angles. We can ideally imagine the overall system described by (6) as constituted of two subsystems, the angular rotations and the linear translations, (Fig. 3)

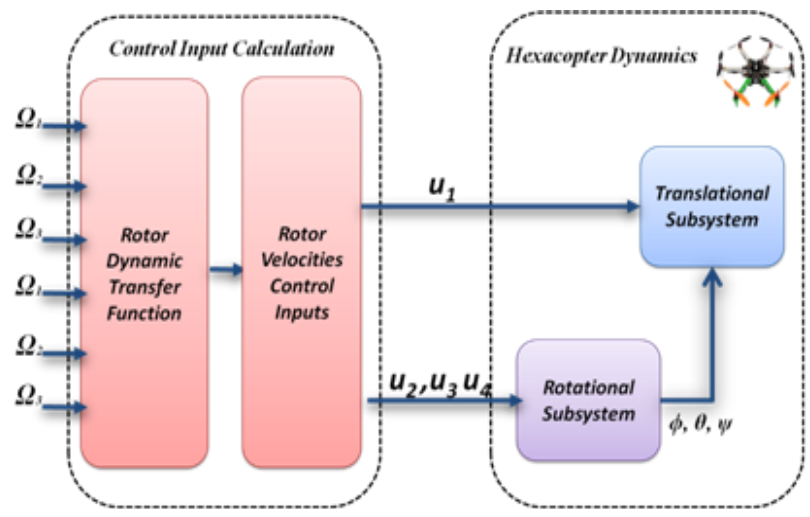

Fig 3: Connection of the two ideal subsystems of the overall dynamical system

\subsubsection{Rotor Dynamics}

The rotors are driven by DC-motors with the well known equations:

$$
\mathrm{L} \frac{d i}{d t}=\mathrm{u}-\mathrm{Ri}-\mathrm{k}_{\mathrm{c}} \omega_{\mathrm{m}} \quad \text { and } \quad J \frac{d \omega_{\mathrm{m}}}{d t}=\tau_{\mathrm{m}}-\tau_{\mathrm{d}} .
$$

As we use a small motor with a very low inductance, the second order DC-motor dynamics may be approximated [5]:

$$
J \frac{d \omega_{m}}{d t}=-\frac{k_{m}^{2}}{R} \omega_{\mathrm{m}}-\tau_{\mathrm{d}}+\frac{k_{m}}{R} \mathrm{u}
$$

By introducing the propeller and the gearbox models, the equation may be rewritten:

$$
\dot{\omega}_{\mathrm{m}}=-\frac{1}{\tau} \omega_{\mathrm{m}}-d \omega_{m}^{2} / \eta r^{3} J_{t}+\frac{1}{k_{m} \tau} \mathrm{u} \quad \text { with } \frac{1}{\tau}=\frac{k_{m}^{2}}{R J_{t}}
$$

The equation can be linearized around an operation point $\dot{\omega}_{0}$ to the form $\dot{\omega}_{\mathrm{m}}=-\mathrm{A} \omega_{\mathrm{m}}+\mathrm{Bu}+\mathrm{C}$.

In this next section, we present the application of two different control techniques Backstepping and Sliding-mode to hexarotor.

\section{CONTROL OF HEXAROTOR}

In this section, a control strategy is based on two loops (inner loop and outer loop). The inner loop contains four control laws: roll command $(\phi)$, pitch command $(\theta)$, yaw control $(\psi)$ and controlling altitude $\mathrm{Z}$. The outer loop includes two control laws positions $(\mathrm{x}, \mathrm{y})$. The outer control loop generates a desired for roll movement $\left(\theta_{d}\right)$ and pitch $\left(\phi_{d}\right)$ through the correction block. This block corrects the rotation of roll and pitch depending on the desired yaw $\left(\psi_{d}\right)$. The figure below shows the control strategy we will adopt Fig.4:

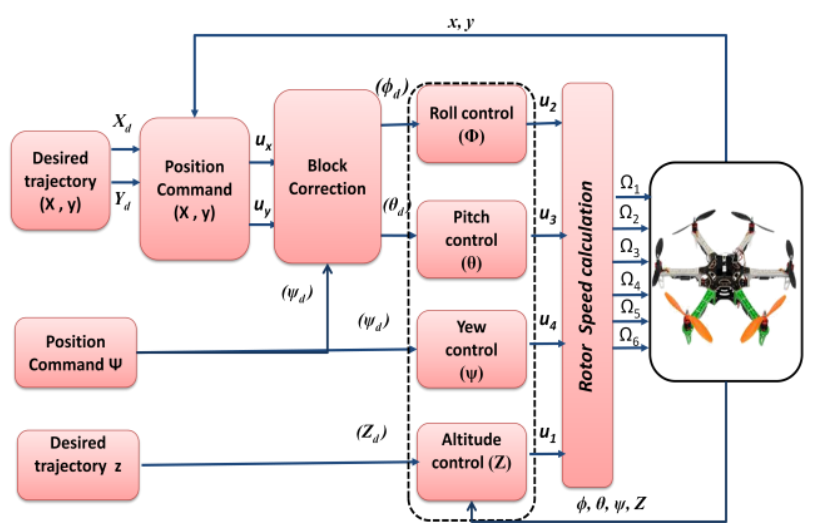

Fig. 4. Synoptic scheme of the proposed control strategy 
The hexarotor parameters used in the simulations are,

Table. 2 Parameters Used in the Simulations

Table. 2 Parameters Used in the Simulations
\begin{tabular}{|l|l|l|}
\hline \multicolumn{1}{|c|}{ Description } & \multicolumn{1}{|c|}{ Value } \\
\hline Pxx & MOI about body frame's x-axis & $7.5 \mathrm{e}-3 \mathrm{~kg} \cdot \mathrm{m} 2$ \\
\hline Jyy & MOI about body frame's y-axis & $7.5 \mathrm{e}-3 \mathrm{~kg} \cdot \mathrm{m} 2$ \\
\hline $\mathrm{Jzz}$ & MOI about body frame's z-axis & $1.3 \mathrm{e}-2 \mathrm{~kg} \cdot \mathrm{m} 2$ \\
\hline 1 & Moment arm & $0.23 \mathrm{~m}$ \\
\hline $\mathrm{Jr}$ & Rotor inertia & $6 \mathrm{e}-5 \mathrm{~kg} . \mathrm{m} 2$ \\
\hline $\mathrm{m}$ & Quadrotor mass & $0.650 \mathrm{~kg}$ \\
\hline $\mathrm{K}_{\mathrm{f}}$ & Aerodynamic force constant & $3.13 \mathrm{e}-5 \mathrm{~N} \mathrm{~s} 2$ \\
\hline $\mathrm{K}_{\mathrm{M}}$ & Aerodynamic moment constant & $7.5 \mathrm{e}-7 \mathrm{Nm} \mathrm{s} 2$ \\
\hline $\mathrm{Rmot}$ & Motor circuit resistance & $0.6 \Omega$ \\
\hline $\mathrm{Kmot}$ & Motor torque constant & $5.2 \mathrm{mNm} / \mathrm{A}$ \\
\hline $\mathrm{Kt}$ & Aerodynamic translation coeff & $\operatorname{diag}(0.1,0.1,0.15)$ \\
\hline $\mathrm{Kt}$ & Aerodynamic rotation coeff & $\operatorname{diag}(0.1,0.1,0.15)$ \\
\hline
\end{tabular}

\subsection{PID Controller for hexarotor}

The classical PID linear controller has the advantage that parameter gains are easy to adjust, is simple to design and has good robustness. However some of the major challenges with the hexarotor include the non-linearity associated with the mathematical model and the imprecise nature of the model due to unmodeled or inaccurate mathematical modeling of some of the dynamics. Therefore applying PID controller to the hexarotor limits its performance.

The purpose of the PID controller is to force the Euler angles to follow desired trajectories. The objective in PID controller design is to adjust the gains to arrive at an acceptable degree of tracking performance in Euler angles.

After the mathematical model of the hexarotor along with its open loop simulation is verified, a PID controller was developed. The PID controller generates the desired control inputs for the hexarotor. The block diagram for a PID controller is shown in Figure 5.

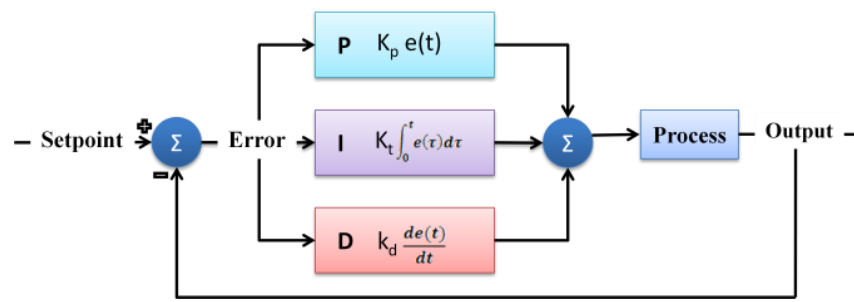

Fig. 5 PID Controller Block Diagram

\subsubsection{Altitude controller}

$U_{l}=k_{p, z}\left(z-z_{d}\right)+k_{d, z}\left(\dot{z}-\dot{z}_{d}\right)+k_{i, z} \int\left(z-z_{d}\right) d t$.

Where

$z_{d}$ and $\dot{z}_{d}$ : Desired altitude and altitude rate of change.

\subsubsection{Attitude controller}

The control objective is to maintain the hexarotor in a constant altitude (z). The PID controller for the $\phi, \theta$ and $\psi$ dynamics can be given as

$$
\begin{array}{ll}
U_{2}=k_{p, \phi}\left(\phi_{d}-\phi\right)+k_{d, \phi}\left(\dot{\phi}_{d}-\dot{\phi}\right)+k_{i, \phi} \int\left(\phi_{d}-\phi\right) d t & (\text { Roll angle }) \\
U_{3}=k_{p, \theta}\left(\theta_{d}-\theta\right)+k_{d, \theta}\left(\dot{\theta}_{d}-\dot{\theta}\right)+k_{i, \theta} \int\left(\theta_{d}-\theta\right) d t & (\text { Pitch angle }) \\
U_{4}=k_{p, \psi}\left(\psi_{d}-\psi\right)+k_{d, \psi}\left(\dot{\psi}_{d}-\dot{\psi}\right)+k_{i, \psi} \int\left(\psi_{d}-\psi\right) d t & (\text { Yaw angle })
\end{array}
$$

Where

$\phi_{d}$ and $\dot{\phi}_{d}$ : Desired roll angle and rate of change $s^{2}$

$\theta_{d}$ and $\dot{\theta}_{d}$ : Desired Pitch angle and rate of change $\psi_{d}$ and $\dot{\psi}_{d}$ : Desired Yaw angle and rate of change

In order to design the PID controllers, nonlinear rotational dynamics of hexarotor are linearized around zero, which are given by,

$\phi(\mathrm{s})=\frac{1}{s^{2}} \frac{l}{I_{x x}} U_{2}(\mathrm{~s}) ; \theta(\mathrm{s})=\frac{1}{s^{2}} \frac{l}{I_{y y}} \mathrm{U}_{3}(\mathrm{~s}) ; \psi(\mathrm{s})=\frac{1}{s^{2}} \frac{l}{I_{z z}} U_{4}(\mathrm{~s})$

\subsubsection{Position controller}

PID controller is defined for controlling the $e_{x}=x_{d}-x$ and $e_{y}=y_{d}-y$ positions. The control objective is to drive both values to zero $\left(e_{x}, e_{x}\right)=(0,0)$. In this sense, the control laws are:

$\ddot{x}_{d}=k_{p, x}\left(x_{d}-x\right)+k_{d, x}\left(\dot{x}_{d}-\dot{x}\right)+k_{i, x} \int\left(x_{d}-x\right) d t$

$\ddot{y}_{d}=k_{p, y}\left(y_{d}-y\right)+k_{d, y}\left(\dot{y}_{d}-\dot{y}\right)+k_{i, y} \int\left(y_{d}-y\right) d t$

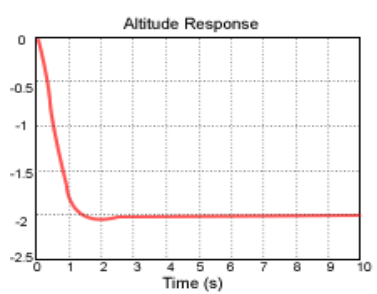
Yaw Angle Respons
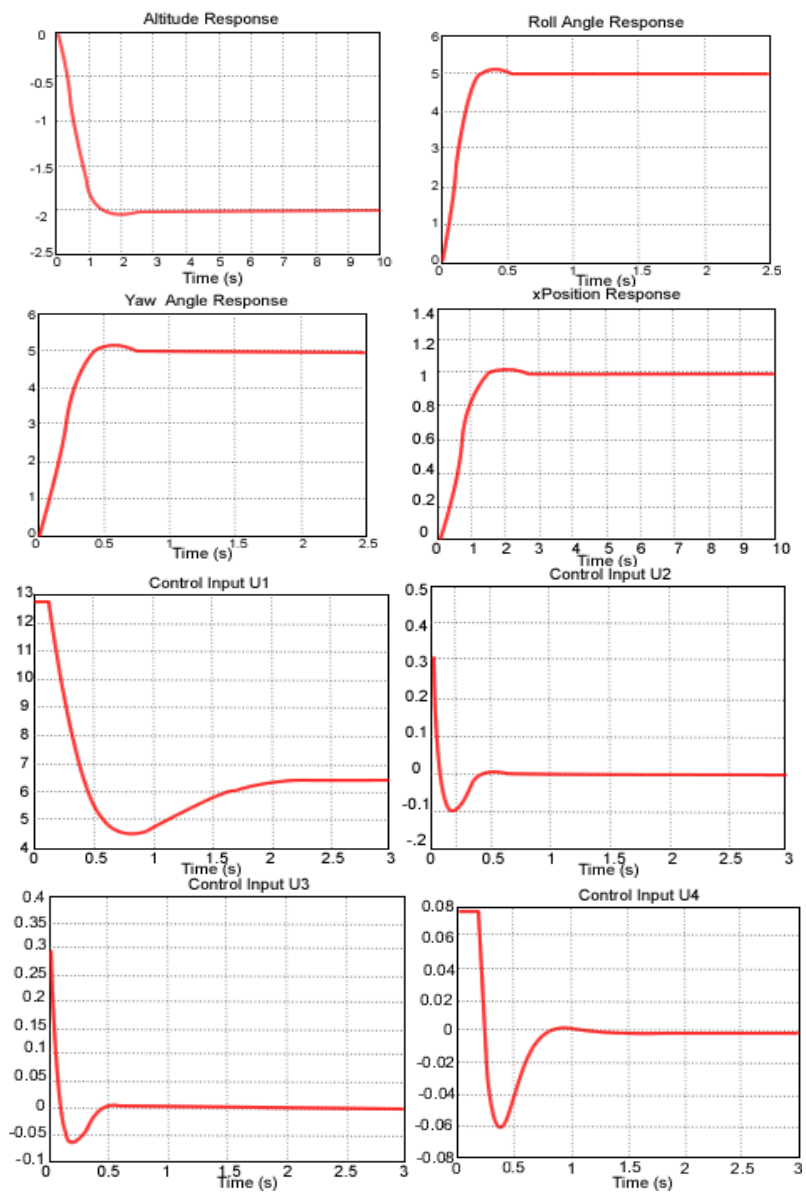

Fig. 6. The PID Control Inputs and Simulation Response

\subsection{Backstepping Controller for Hexarotor}

Backstepping design refers to "step back" to the control input, and a major advantage of backstepping design is its flexibility to avoid cancellation of useful nonlinearities and pursue the objectives of stabilization and tracking, rather than those of linearization. Recursively constructed backstepping controller employs the control Lyapunov function (CLF) to guarantee the global stability [10], [11].

In this section, a Backstepping controller is used to control the attitude, heading and altitude of the hexarotor. The Backstepping controller is based on the state space model derived in (7). Using the backstepping approach, one can synthesize the control law forcing the system to follow the desired trajectory. Refer to [6] 
and [7] for more details.

Considering the following system,

$$
e_{i}=\left\{\begin{array}{cc}
x_{i d}-x_{i} & i \in[3,5,7,9,11] \\
x_{(i-1) d}+k_{(i-1)} e_{(i-1)}-x_{i} & i \in[4,6,8,10,12] \\
\text { with } k_{i}>0 & i \in[2, \ldots ., 12]
\end{array}\right.
$$

\subsubsection{Backstepping Control of the Rotations}

\section{Subsystem}

Using the backstepping approach, one can synthesize the control law forcing the system to follow the desired trajectory. Refer to [7] and [8] for more details. For the first step we consider the tracking-error $e_{i}=x_{I d}-x_{1}$ and we use the Lyapunov theorem by considering the Lyapunov function $V_{i}$ positive definite and it's time derivative negative semi-definite:

$$
V_{i}= \begin{cases}\frac{1}{2} e_{i}^{2} & i \in[3,5,7,9,11] \\ V_{i-1}+\frac{1}{2} e_{i}^{2} & i \in[4,6,8,10,12]\end{cases}
$$

For the first step we consider the tracking-error:

$$
\left\{\begin{array}{l}
e_{1}=x_{l d}-x_{1}=\phi_{d}-x_{1} \\
V_{l}=\frac{1}{2} e_{1}^{2} \quad \text { and } \quad \dot{V}_{l}=e_{1} \dot{e}_{l}=e_{l}\left(\dot{\phi}_{d}-x_{2}\right)
\end{array}\right.
$$

The stabilization of $e_{l}$ can be obtained by introducing a virtual control input $\mathrm{x}_{2}$ :

$$
\mathrm{x}_{2}=\dot{\phi}_{d}+\mathrm{k}_{1} e_{1} \Longrightarrow \dot{V}_{1}=-\mathrm{k}_{1} e_{1}^{2} \leq 0 .
$$

For the second step we consider the augmented Lyapunov function:

$$
\left\{\begin{array}{l}
e_{2}=\dot{\phi}_{d}+\mathrm{k}_{1} e_{1}-x_{2} \\
V_{2}=\frac{1}{2} e_{1}^{2}+\frac{1}{2} e_{2}^{2}
\end{array}\right.
$$

And it's time derivative is then: $\quad \dot{V}_{2}=e_{1} \dot{e}_{1}+e_{2} \dot{e}_{2}$

$\dot{V}_{2}==e_{1}\left(-k_{1} e_{1}+e_{2}\right)+e_{2}\left(\ddot{\phi}_{d}+k_{1} \dot{e}_{1}-\mathrm{a}_{1} \mathrm{x}_{4} \mathrm{x}_{6}-\mathrm{a}_{2} x_{2}^{2}-\mathrm{a}_{3} \Omega_{\mathrm{r}} \mathrm{x}_{4}-\mathrm{b}_{1}\right.$ $\mathrm{u}_{2}$ )

The control input $\mathrm{U} 2$ is then extracted, satisfying:

$$
\dot{V}_{2}=-k_{1} e_{1}^{2}-k_{2} e_{2}^{2} \leq 0 .
$$

$\mathrm{U}_{2}=\frac{1}{b 1}\left[-\mathrm{a}_{1} \mathrm{x}_{4} \mathrm{x}_{6}-\mathrm{a}_{2} x_{2}^{2}-\mathrm{a}_{3} \Omega_{\mathrm{r}} \mathrm{x}_{4}+\ddot{\phi}_{d}+k_{l}\left(-k_{1} e_{1}+e_{2}\right)_{+} k_{2} e_{2}+e_{I}\right]$

Following exactly the same steps as the roll controller, the control input $\mathrm{U}_{3}$ responsible of generating the pitch rotation and $\mathrm{U}_{4}$ responsible of generating the yaw rotation are calculated to be,

\section{Roll controller}

$\mathrm{U}_{2}=\frac{1}{b 1}\left[-\mathrm{a}_{1} \mathrm{x}_{4} \mathrm{x}_{6}-\mathrm{a}_{2} x_{2}^{2}-\mathrm{a}_{3} \Omega_{\mathrm{r}} \mathrm{x}_{4}+\ddot{\phi}_{d}+k_{l}\left(-k_{l} e_{1}+e_{2}\right)_{+} k_{2} e_{2}+\right.$ $\left.e_{1}\right]$

\section{Pitch controller}

$$
\begin{aligned}
& \mathrm{U}_{3}=\frac{1}{b 2}\left[-\mathrm{a}_{4} \mathrm{x}_{2} \mathrm{x}_{6}-\mathrm{a}_{5} x_{4}^{2}-\mathrm{a}_{6} \Omega_{\mathrm{r}} \mathrm{x}_{2}+\ddot{\theta}_{d}+k_{3}\left(-k_{3} e_{3}+e_{4}\right)_{+} k_{4} e_{4}+\right. \\
& \left.e_{3}\right]
\end{aligned}
$$

$$
\begin{aligned}
& \text { Yaw controller } \\
& \mathrm{U}_{4}=\frac{1}{b 3}\left[-\mathrm{a}_{7} \mathrm{x}_{2} \mathrm{x}_{4}-\mathrm{a}_{8} x_{6}^{2}+\ddot{\psi}_{d}+k_{5}\left(-k_{5} e_{5}+e_{6}\right)_{+} k_{6} e_{6}+e_{5}\right]
\end{aligned}
$$

\subsubsection{Backstepping Control of the Linear} Translations

The altitude control U1 and the Linear $\left(\mathrm{u}_{\mathrm{x}}, \mathrm{u}_{\mathrm{y}}\right)$ Motion Control are obtained using the same approach described in 3.2.

$$
\begin{aligned}
& \mathrm{U}_{1}=\frac{m}{\operatorname{coss} 1 \cos \mathrm{x} 3}\left[\mathrm{~g}-\mathrm{a}_{11} \mathrm{x}_{12}+\ddot{z}_{\mathrm{d}}+k_{11}\left(-k_{11} e_{11}+e_{12}\right)_{+} k_{12} e_{12}+e_{11}\right] \\
& \mathrm{u}_{\mathrm{x}}=\left(\frac{m}{u_{1}}\right)\left[-\mathrm{a}_{9} \mathrm{x}_{8}+\ddot{x}_{\mathrm{d}}+k_{7}\left(-k_{7} e_{7}+e_{8}\right)_{+} k_{8} e_{8}+e_{7}\right] \\
& \mathrm{u}_{\mathrm{y}}=\left(\frac{m}{u_{1}}\right)\left[-\mathrm{a}_{10} \mathrm{x}_{10}+\ddot{y}_{\mathrm{d}}+k_{9}\left(-k_{9} e_{9}+e_{10}\right)_{+} k_{10} e_{10}+e_{9}\right] .
\end{aligned}
$$

The sliding mode control inputs which were derived and expressed in equation (8) were applied to the nonlinear model in (7) and responses are shown in fig.(7).
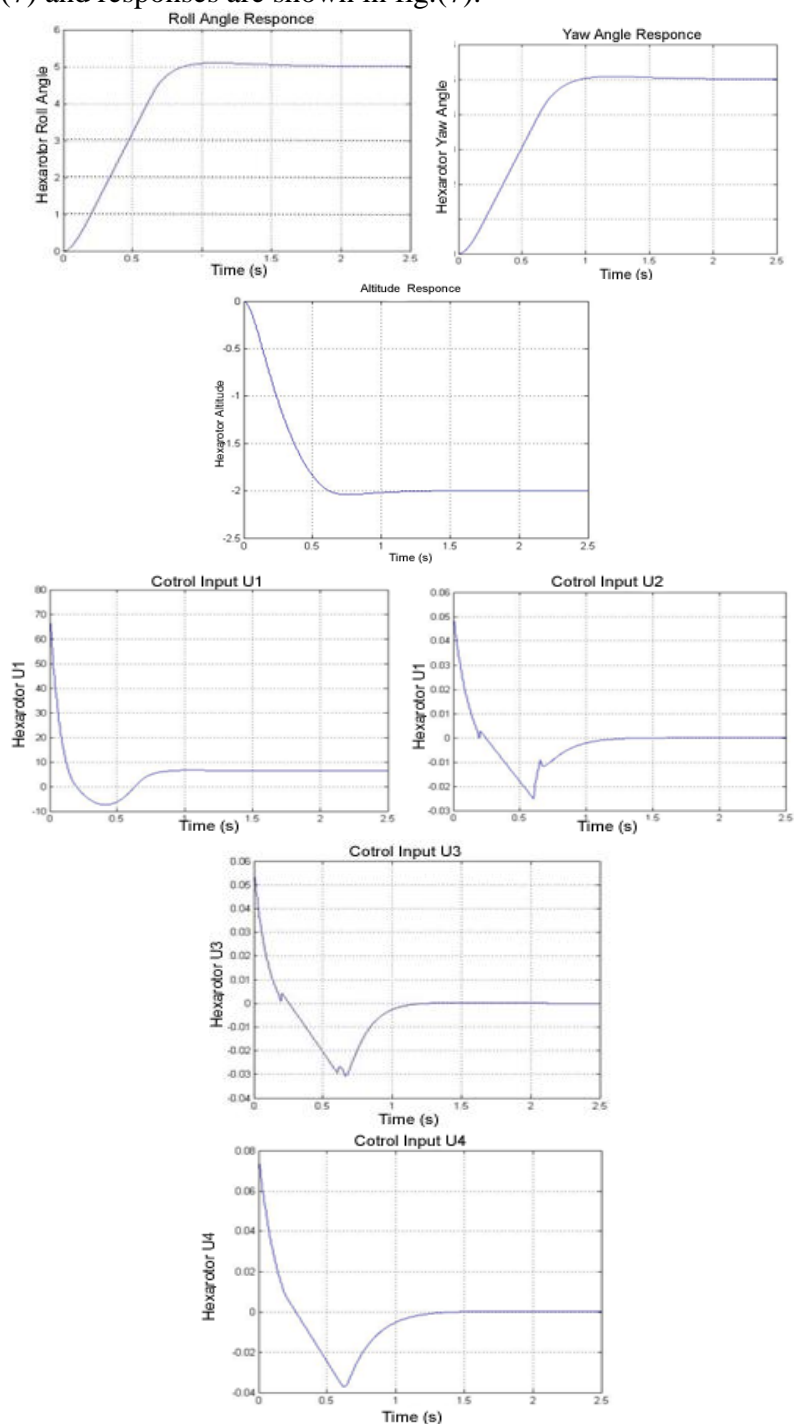

Fig. 7. The backstepping control inputs and simulation response

\subsection{Sliding-Mode Control of Hexarotor}

Sliding mode control is a well-established nonlinear control technique that displays certain degree of robustness against uncertainties and disturbances. Its main advantage is that it does not simplify the dynamics through linearization and has good tracking. Although it is vulnerable to noise and it suffers from chattering phenomenon, several approaches have been proposed to overcome these difficulties without giving concessions from the robustness property of the scheme. The behavior is composed of two phases, first the error dynamics is guided toward a predefined subspace of the state space, which we call 
the sliding manifold, and the behavior during this phase is called the reaching phase. This phase is known for the high magnitude control efforts that may undergo saturation sometimes. Second is the sliding phase, during which the error vector obeys the dynamical implications of the analytic description of the locus itself, which is stable by design and the error terminates at the origin inevitably. The control scheme takes its name from the latter dynamic behavior and is called sliding mode control.

The hexarotor system was subdivided into the full-actuated and under-actuated systems. The under-actuated system, to which SMC was applied, was further subdivided into under-actuated subsystems. Results showed good stability and robustness of the system. Chattering effect of SMC was observed but minimized with a continuous approximation of a pre-determined "sign" function

The basic sliding mode controller design procedure is performed in two steps. Firstly, choice of sliding surface (S) is made according to the tracking error, while the second step consist the design of Lyapunov function which can satisfy the necessary sliding condition $(S \dot{S}<0)$ [9][10]. The application of sliding mode control to hexarotor dynamic is presented here by obtaining the expression for control input. The sliding surface are define,

$$
\begin{aligned}
& S_{\phi}=\mathrm{e}_{2}+\lambda_{\phi} \mathrm{e}_{1}=\dot{x}_{1 \mathrm{~d}}-\mathrm{x}_{2}+\lambda_{\phi}\left(\mathrm{x}_{1 \mathrm{~d}}-\mathrm{x}_{1}\right) \\
& \mathrm{S}_{\theta}=\mathrm{e}_{4}+\lambda_{\theta} \mathrm{e}_{3}=\dot{x}_{3 \mathrm{~d}}-\mathrm{x}_{4}+\lambda_{\theta}\left(\mathrm{x}_{3 \mathrm{~d}}-\mathrm{x}_{3}\right) \\
& \mathrm{S}_{\psi}=\mathrm{e}_{6}+\lambda_{\psi} \mathrm{e}_{5}=\dot{x}_{5 \mathrm{~d}}-\mathrm{x}_{6}+\lambda_{\psi}\left(\mathrm{x}_{5 \mathrm{~d}}-\mathrm{x}_{5}\right) \\
& \mathrm{S}_{x}=\mathrm{e}_{8}+\lambda_{\mathrm{x}} \mathrm{e}_{7}=\dot{x}_{7 \mathrm{~d}}-\mathrm{x}_{8}+\lambda_{\mathrm{x}}\left(\mathrm{x}_{7 \mathrm{~d}}-\mathrm{x}_{7}\right) \\
& \mathrm{S}_{\mathrm{y}}=\mathrm{e}_{10}+\lambda_{\mathrm{y}} \mathrm{e}_{9}=\dot{x}_{9 \mathrm{~d}}-\mathrm{x}_{10}+\lambda_{\mathrm{y}}\left(\mathrm{x}_{9 \mathrm{~d}}-\mathrm{x}_{9}\right) \\
& \mathrm{S}_{\mathrm{z}}=\mathrm{e}_{12}+\lambda_{\mathrm{z}} \mathrm{e}_{11}=\dot{x}_{11 \mathrm{~d}}-\mathrm{x}_{12}+\lambda_{\mathrm{z}}\left(\mathrm{x}_{11 \mathrm{~d}}-\mathrm{x}_{11}\right)
\end{aligned}
$$

Such that

$$
\left\{\begin{array}{l}
\mathrm{e}_{i}=\mathrm{x}_{i d}-\mathrm{x}_{i} \\
\mathrm{e}_{i+1}=\dot{e}_{i} \\
\lambda_{i}>0
\end{array} \quad i \in[1, \ldots, 11]\right.
$$

Assuming here that $V\left(S_{\phi}\right)=\frac{1}{2} S_{\phi}^{2}$ then, the necessary sliding condition is verified and lyapunov stability is guaranteed. The chosen law for the attractive surface is the time derivative of satisfying $(S \dot{S}<0)$

$$
\begin{aligned}
\dot{S}_{\phi}= & -k_{\phi} \operatorname{sign}\left(S_{\phi}\right) \\
& =\dot{e}_{2}+\lambda_{\phi} \dot{e}_{1}=\ddot{x}_{1 d}-\dot{x}_{2}+\lambda_{\phi}\left(\dot{x}_{1 \mathrm{~d}}-\mathrm{x}_{2}\right) \\
& =-a_{1} \mathrm{x}_{4} \mathrm{x}_{6}-a_{2} \mathrm{x}_{4} \Omega_{\mathrm{r}}-\mathrm{b}_{1} \mathrm{U}_{2}+\ddot{\phi}_{\mathrm{d}}+\lambda_{\phi}\left(\dot{\phi}_{\mathrm{d}}-\mathrm{x}_{2}\right) . \\
\mathrm{U}_{2}= & \frac{1}{\mathrm{~b} 1}\left[-a_{1} \mathrm{x}_{4} \mathrm{x}_{6}-a_{2} \mathrm{x}_{4} \Omega_{\mathrm{r}}+\ddot{\phi}_{\mathrm{d}}+\lambda_{\phi}\left(\dot{\phi}_{\mathrm{d}}-\mathrm{x}_{2}\right)-k_{\phi} \operatorname{sign}\left(\mathrm{S}_{\phi}\right)\right] .
\end{aligned}
$$

The same steeps are followed to extract $\mathrm{U}_{3}, \mathrm{U}_{4}$ and $\mathrm{U}_{1}$ :

$$
\begin{array}{ll}
\mathrm{U}_{2}=\frac{1}{\mathrm{~b} 1}\left[-a_{1} \mathrm{x}_{4} \mathrm{x}_{6}-a_{2} \mathrm{x}_{4} \Omega_{\mathrm{r}}+\ddot{\phi}_{\mathrm{d}}+\lambda_{\phi} \mathrm{e}_{2}-k_{\phi} \operatorname{sign}\left(\mathrm{S}_{\phi}\right)\right] & (\text { Roll }) \\
\mathrm{U}_{3}=\frac{1}{\mathrm{~b} 2}\left[-a_{3} \mathrm{x}_{2} \mathrm{x}_{6}-a_{4} \mathrm{x}_{2} \Omega_{\mathrm{r}}+\ddot{\theta}_{d}+\lambda_{\theta} \mathrm{e}_{4}-k_{\theta} \operatorname{sign}\left(\mathrm{S}_{\theta}\right)\right] \quad(\mathrm{Pitch}) \\
\mathrm{U}_{4}=\frac{1}{\mathrm{~b} 3}\left[-a_{5} \mathrm{x}_{2} \mathrm{x}_{4}-a_{4} \mathrm{x}_{2} \Omega_{\mathrm{r}}+\ddot{\psi}_{d}+\lambda_{\psi} \mathrm{e}_{6}-k_{\psi} \operatorname{sign}\left(\mathrm{S}_{\psi}\right)\right] \quad(\text { Yaw) } \\
\mathrm{U}_{1}=\frac{m}{\cos 1 \cos \mathrm{x} 3}\left[g+\ddot{z}_{d}+\lambda_{\mathrm{z}} \mathrm{e}_{8}-k_{z} \operatorname{sign}\left(\mathrm{S}_{\mathrm{z}}\right)\right] \\
\mathrm{u}_{\mathrm{x}}=\left(\frac{m}{u_{1}}\right)\left[\ddot{x}_{d}+\lambda_{\mathrm{x}} \mathrm{e}_{10}-k_{x} \operatorname{sign}\left(\mathrm{S}_{\mathrm{x}}\right)\right] \\
\mathrm{u}_{\mathrm{y}}=\left(\frac{m}{u_{1}}\right)\left[\ddot{y}_{d}+\lambda_{\mathrm{y}} \mathrm{e}_{12}-k_{y} \operatorname{sign}\left(\mathrm{S}_{\mathrm{y}}\right)\right] & \text { (Altitude) }
\end{array}
$$

The sliding mode control inputs which were derived and expressed in equation (9) were applied to the nonlinear model in (7) and responses are shown in fig. 9 and fig. 10.
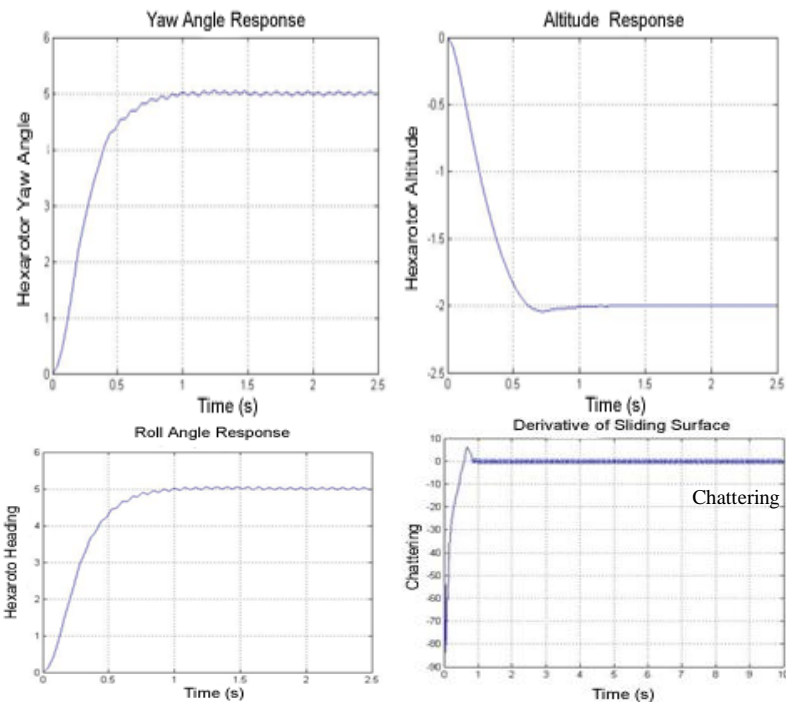

Fig. 9 The sliding Control Simulation Response
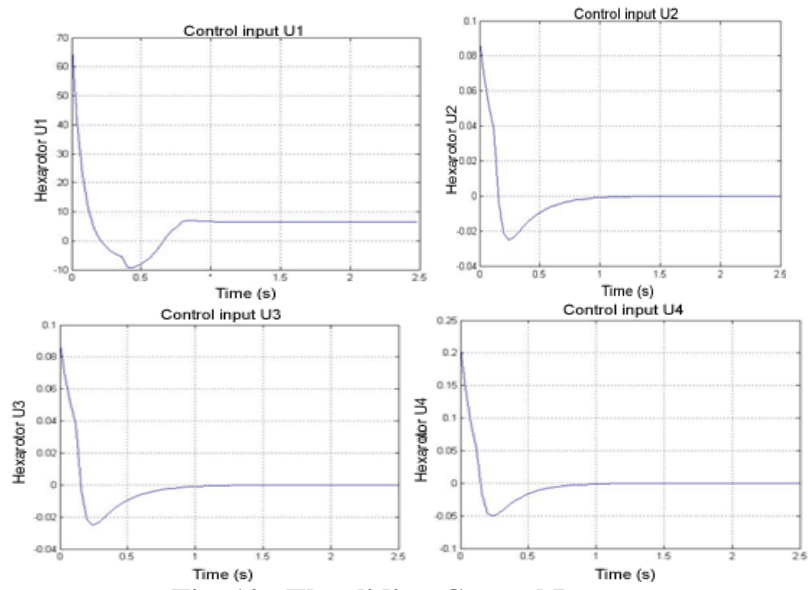

Fig. 10. The sliding Control Inputs

\section{RESULTS AND DISCUTION}

To be able to compare fairly between the three implemented control techniques, the response graph of the system under the effect of each the three controllers was plotted superimposed on one another. Figure. 11 show the altitude response, the attitude and heading responses respectively.
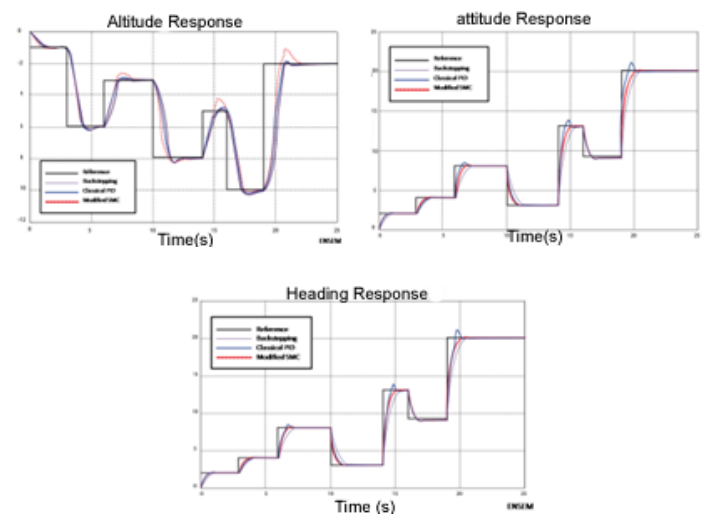

Fig. 11 the altitude, the attitude and heading responses

\subsection{Linear Operation}

In this paper three linear and nonlinear control schemes are used to stabilize the attitude of hexarotor UAV. The methods considered are PID, SMC, backstepping control. The employed controllers developed to control the hexarotor model under 
consideration gave comparable dynamic performances in terms of settling time and overshoot when they were deployed in near hover stabilization of the hexarotor.

The PID controller has been successfully applied to the hexarotor though with some limitations. The tuning of the PID controller could pose some challenges as this must be conducted around the equilibrium point, which is the hover point, to give good performance.

The SMC resulted in good stability and robustness of the system; but an undesirable Chattering effect of SMC was observed which was very notable in the attitude response unlike the altitude. It minimized with a continuous approximation of a predetermined "sign" function. The presence of the "sign" term in the SMC's control law makes it a discontinuous controller. Shows that whenever the value of the surface $s$ is positive, the control law works to decrease the trajectory to reach the sliding surface $(s=0)$. Ideally it should continue sliding on the surface once hitting it, but due to the delay between the change of sign and the change in the control action, the trajectory passes the surface to the other side. The main drawbacks of chattering are that it causes the excitation of unmodeled system dynamics that yields a possible instability of the system. In addition to that it is associated with a high power consumption and possible actuator damage. These drawbacks make the SMC hard to be implemented on real systems. Backstepping control is a recursive algorithm that breaks down the controller into steps and progressively stabilizes each subsystem. Its advantage is that the algorithm converges fast leading to less computational resources and it can handle disturbances well. The main limitation with the algorithm is its robustness is not good. To increase robustness (to external disturbances) of the general backstepping algorithm, an integrator is added and the algorithm becomes Integrator backstepping control. The integral approach was shown to eliminate the steady-state errors of the system, reduce response time and restrain overshoot of the control parameters. Figure 11 show a quantitative comparison between the performance of the PID, SMC and Backstepping controllers in terms of the settling time and overshoot of the system's response respectively.

\subsection{Nonlinear Operation}

When the controllers were used outside of the linear region (away from hover), the PID controller failed to stabilize the system due to the fact that PID comes out of a family of linear controllers. On the other hand, the SMC and the Backstepping controller were able to stabilize the system with a good dynamic performance.

In conclusion, PID controller is simple in structure; its performance may not be adequate under conditions of severe disturbances. SMC is robust against disturbances yet it suffers from the chattering phenomenon. Backstepping yield good tracking results yet they need the availability of the system nonlinearities, which is generally accepted as a drawback. In table. 3 summarizes the comparison of the various algorithms as applied to hexarotors. The performance of a particular algorithm depends on many factors that may not even be modeled. Hence, this table serves as guide in accordance with what is presented in this work.

Table.3 Comparison of control algorithms (PID, SMC, BS)

$\begin{array}{lccr}\text { Optimal } & \text { LN } & \text { A } & \text { LN } \\ \text { Intelligent } & \text { LN } & \text { LN } & \text { LN } \\ \text { Tracking ability } & \text { A } & \text { H } & \text { H } \\ \text { Fast convergence } & \text { A } & \text { H } & \text { LN } \\ \text { Precision } & \text { A } & \text { H } & \text { A } \\ \text { Simplicity } & \text { H } & \text { A } & \text { LN } \\ \text { Disturbance rejection } & \text { LN } & \text { H } & \text { H } \\ \text { Noise (signal) } & \text { H } & \text { LN } & \text { LN } \\ \text { Chattering } & \text { LN } & \text { H } & \text { LN }\end{array}$

Legend: $\mathbf{L N}$ - low to none; $\mathbf{A}$-average; $\mathbf{H}$ - high.

\section{CONCLUSIONS AND FUTURE WORKS}

The goal of this work was to derive a mathematical model for the hexarotor Unmanned Aerial Vehicle (UAV) and develop nonlinear control algorithms to stabilize the states of the hexarotor, which include its altitude, attitude, heading and position in space and to verify the performance of these controllers with comparisons via computer simulations. The mathematical model of a hexarotor UAV was developed in details including its aerodynamic effects and rotor dynamics which we found lacking in many literatures; three control techniques were then developed and synthesized; a linear Proportional-Integral-Derivative (PID) controller, a nonlinear Sliding Mode Controller (SMC) and a nonlinear Backstepping controller. A complete simulation was then implemented on MATLAB/Simulink relying on the derived mathematical model of the hexarotor. The simulation environment was used to evaluate the mentioned controllers and compare their dynamic performances under different types of input conditions. The SMC and Backstepping controllers gave better performance outside the linear hovering region due to their nonlinear nature. The PID and Backstepping controllers gave better performance than all the other controllers when the effect of wind was added to the system. The wind effect was modeled as extra forces and moments on the hexarotor body.

As it can be seen from the experimental plots, the controller introduced using the sliding-mode approach provides average results. This is partly due to switching nature of the controller which introduces high frequency, low amplitude vibrations causing the sensor to drift. On the other hand, the backstepping controller proves the ability to control the orientation angles in the presence of relatively high perturbations confirming by the way some previous studies on under actuated systems. The SMC controller has the problem of chattering.

An important part of this work was dedicated to finding a good control approach for hexarotors. Three techniques were explored from theoretical development to final experiments.

As evident from the review, no single algorithm presents the best of the required features. It also been discussed that getting the best performance usually requires hybrid control schemes that have the best combination of robustness, adaptability, optimality, simplicity, tracking ability, fast response and disturbance rejection among other factors.

The future work is to develop a fully autonomous vehicle with a more powerful hybrid flight controller. For example, adding an integral action to the developed Backstepping controller combined with Frenet-Serret Theory will lead to the formulation of an adaptive control algorithm robust to system uncertainties. Last but not least, implementing the developed control 
techniques on real hexarotor hardware to give a more fair comparison between their performances. The positive results achieved through this development enhance the knowledge of this very unstable system and encourages us to continue towards full autonomy hexarotor.

\section{REFERENCES}

[1] Hongning Hou, Jian Zhuang, Hu Xia, Guanwei Wang, and Dehong $\mathrm{Yu}$. A simple controller of minisize quad-rotor vehicle. In Mechatronics and Automation (ICMA), 2010 International Conference on, pages 1701doi: 10.1109/ICMA.2010.5588802.

[2] Jinhyun Kim, Min-Sung Kang, and Sangdeok Park. Accurate modeling and robust hovering control for a quadrotor vtol aircraft. Journal of Intelligent and Robotic Systems, 57(1-4):9-26, 2010.

[3] Farid Kendoul. Survey of advances in guidance, navigation, and control of unmanned rotorcraft systems. Journal of Field Robotics, 29(2):315\{378, 2012.

[4] Simon János, Goran Martinovic, Navigation of Mobile Robots Using WSN`s RSSI Parameter and Potential Field Method, Acta Polytechnica Hungarica, Journal of Applied Sciences Vol.10, No.4, pp. 107-118, 2013.
[5] Jun Shen, Qiang Wu, Xuwen Li, Yanhua Zhang, Research of the RealTime Performance of Operating System, 5th International conference on Wireless Communications, $\mathrm{pp}$. $1-4,2009$.

[6] R. M. Murray and S. S. Sastry, A mathematical introduction to robotic manip-ulation. CRC press, 1994

[7] A.W.A. Saif, M. Dhaifullah, M.A.Malki and M.E. Shafie, "Modiffied Backstepping Control of Quadrotor", International Multi-Conference on System, Signal and Devices, 2012.

[8] A.A. Main, W. Daobo, "Modeling and Backstepping-based Nonlinear Control Strategy for a 6 DOF Quadrotor Helicopter", Chinese Journal of Aeronautics 21, pp. 261268, 2008.

[9] V.G. Adir, A.M.Stoica and J.F. Whidborne,"Sliding Mode Control of 4Y Octorotor", U.P.B. Sci. Bull, Series D, Vol.74, Iss. 4, pp. 37-51,2012.

[10] K. Runcharoon and V. Srichatrapimuk, "Sliding Mode Control of Quadrotor" International Conference of Technological Advances in Electrical, Electronics and Computer Engineering, pp. 552-556 May 9-11, 2013. 